FedUni ResearchOnline

https://researchonline.federation.edu.

au

This is the author's version of a work that was accepted for publication in the Communications in Mathematical Sciences,16(5), p. 1225-1240. Changes resulting from the publishing process, such as peer review, editing, corrections, structural formatting, and other quality control mechanisms may not be reflected in this document.

Copyright $@ 2018$, International Press of Boston, Inc. All rights reserved. 


\title{
ON SDP METHOD FOR SOLVING CANONICAL DUAL PROBLEM IN POST BUCKLING OF LARGE DEFORMED ELASTIC BEAM*
}

\author{
ELAF JAAFAR ALI ${ }^{\dagger}$ AND DAVID YANG GAO ${ }^{\ddagger}$
}

\begin{abstract}
This paper presents a new methodology and algorithm for solving post buckling problems of a large deformed elastic beam. The total potential energy of this beam is a nonconvex functional, which can be used to model both pre- and post-buckling phenomena. By using a canonical dual finite element method, a new primal-dual semi-definite programming (PD-SDP) algorithm is presented, which can be used to obtain all possible post-buckled solutions. Applications are illustrated by several numerical examples with different boundary conditions. We find that the global minimum solution of the nonconvex potential leads to a stable configuration of the buckled beam, the local maximum solution leads to the unbuckled state, and both of these two solutions are numerically stable. However, the local minimum solution leads to an unstable buckled state, which is very sensitive to axial compressive forces, thickness of beam, numerical precision, and the size of finite elements. The method and algorithm proposed in this paper can be used for solving general nonconvex variational problems in engineering and sciences.
\end{abstract}

Keywords. Post buckling; Nonlinear Gao beam; Canonical dual finite element method; Global optimization; Triality theory.

AMS subject classifications. 93B40.

\section{Introduction}

It is known that the total potential energy for the post-buckling of large deformed structures must be nonconvex to allow multiple local minimum solutions for all possible buckled status [6]. However, nonconvex variational problems have always presented serious challenges not only in computational mechanics, but also in mathematical analysis and computer science [15]. Traditional finite element methods for solving any nonconvex variational problem usually end up with a nonconvex minimization problem in $\mathbb{R}^{n}$. Due to the lack of global optimality criteria, popular nonlinear programming methods developed from convex optimization can't be used to find global optimal solutions. It was discovered in [13] that for certain external loads, both global and local minimum solutions to large deformed mechanics problems are usually nonsmooth and can't be captured by any Newton-type methods. Therefore, most nonconvex optimization problems are considered as NP-hard (Non-deterministic Polynomial-time hard) in computer science. Unfortunately, these well-known difficulties are not fully recognized in computational mechanics due to the significant gap between engineering mechanics and global optimization. Indeed, engineers and scientists are mistakenly attempting to use traditional finite element methods and commercial softwares for solving nonconvex mechanics problems.

Canonical duality theory is a newly developed and potentially powerful methodology which can be used not only for modeling complex systems within a unified framework, but also for solving a large class of challenging problems in nonconvex, nonsmooth, and discrete systems [15]. This theory comprises mainly three parts: 1) a canonical dual transformation, which can be used to formulate perfect dual problem without a duality

\footnotetext{
*Received: November 30, 2017; Accepted (in revised form): March 19, 2018. Communicated by Wotao Yin.

${ }^{\dagger}$ Faculty of Science and Technology, Federation University Australia, Mt Helen, Victoria, Australia \& University of Basrah, College of Science, Basra, Iraq (elafali@students.federation.edu.au).

${ }^{\ddagger}$ Faculty of Science and Technology, Federation University Australia, Mt Helen, Victoria, Australia (d.gao@federation.edu.au).
} 
gap; 2) a complementary-dual variational principle, which presents a unified analytic solution form for general problems in continuous and discrete systems; 3) a triality theory, which can be used to identify both global and local extrema and to develop effective algorithms for solving nonconvex optimization problems.

The canonical duality theory was developed from Gao and Strang's original work on nonconvex/nonsmooth variational/boundary value problems in finite deformation systems [18]. In order to recover the complementary energy principle in nonconvex analysis, they discovered a so-called complementary gap function, which leads to a complementary-dual variational principle in finite deformation mechanics. They proved that the positivity of this gap function provides a global optimality condition for nonconvex variational problem. It was realized seven years later that the negativity of this gap function can be used to identify the biggest local minimal and local maximal solutions. Therefore, a triality theory was first proposed in post-buckling problems of a large deformation beam model [7], and a pure complementary energy principle was obtained in 1999 [8]. This principle solved an open problem in nonlinear elasticity [23], which can be used for obtaining analytical solutions to general large deformation problems $[9,12,15]$. Based on the canonical duality theory and the mixed finite element method, a canonical dual finite element method has been developed [5] with the successful application for solving nonconvex mechanics problems in phase transitions of solids [20]. It was discovered recently $[3,30]$ that the nonconvex variational problem of a post-buckled nonlinear Gao beam can have at most three smooth solutions: a global minimizer representing a stable buckled state, a local maximizer for an unbuckled state, and a local minimizer for an unstable buckled state. Both global minimum and local maximum solutions can be obtained easily by using the canonical dual finite element method. However, the local minimum solution is very sensitive and difficult to obtain by standard convex minimization algorithms.

The main goal of the present paper is to develop a new canonical primal-dual algorithm for solving the post-buckling problem with special attention to the local unstable buckled configuration of a large deformed beam. The generalized total complementary energy associated with this model is a nonconvex functional and is reformulated as a global optimization problem to study the post-buckling responses of the beams. Based on the canonical duality theory and the associated triality theorem, a new primal-dual semi-definite program (PD-SDP) algorithm is proposed for solving this challenging problem to obtain all possible solutions. Applications are illustrated by different boundary value problems. An important mistake in [3] on the local minimum solution is found.

\section{Nonconvex problem and canonical duality theory}

Let us consider an elastic beam subjected to a vertical distributed lateral load $q(x)$ and compressive external axial force $F$ at the right end as shown in Figure 2.1. It was discovered by Gao in 1996 [6] that the well-known von Karman nonlinear plate model in one-dimension is equivalent to a linear differential equation, therefore, it can't be used to study post-buckling phenomena. The main reason for this "paradox" is due to the fact that the stress in the lateral direction of a large deformed plate was ignored by von Karman. Therefore, the von Karman equation works only for thinplates and can't be used as a beam model. For a relatively thick beam such that $h / L \sim w(x) \in O(1)$, the deformation in the lateral direction can't be ignored. Based on the finite deformation theory for Hooke's material and the Euler-Bernoulli hypothesis (i.e. straight lines normal to the mid-surface remain straight and normal to the midsurface after deformation), a nonlinear beam model was proposed by Gao [6]:

$$
E I w_{, x x x x}-\alpha E w_{, x}^{2} w_{, x x}+E \lambda w_{, x x}-f(x)=0, \forall x \in[0, L]
$$




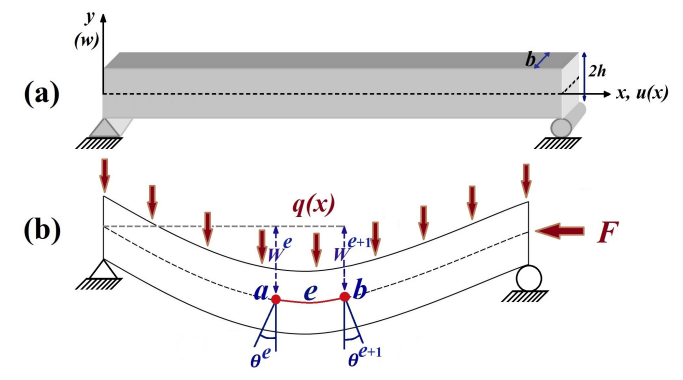

FIG. 2.1. Simply supported beam model - pre and post buckling analysis

where $E$ is the elastic modulus of material, $I=2 h^{3} / 3$ is the second moment of area of the beam's cross-section, $w$ is the transverse displacement field of the beam, $\alpha=$ $3 h\left(1-\mu^{2}\right)>0$ with $\mu$ as the Poisson's ratio, $\lambda=(1+\mu)\left(1-\mu^{2}\right) F / E>0$ is an integral constant, $f(x)=\left(1-\mu^{2}\right) q(x)$ depends mainly on the distributed lateral load $q(x), 2 h$ and $L$ represent the height and length of the beam, respectively. The axial displacement $u(x)$ is governed by the following differential equation [6]:

$$
u_{, x}=-\frac{1}{2}(1+\mu) w_{, x}^{2}-\frac{\lambda}{2 h(1+\mu)},
$$

which shows that if $u(x) \sim w_{, x}(x) \in O(\epsilon)$, then $u_{, x}(x) \sim w_{, x x}(x) \in O\left(\epsilon^{2}\right)$.

The total potential energy of this beam model is $\Pi(w): \mathcal{U}_{a} \rightarrow \mathbb{R}$ defined by

$$
\Pi(w)=\int_{0}^{L}\left(\frac{1}{2} E I w_{, x x}^{2}+\frac{1}{12} E \alpha w_{, x}^{4}-\frac{1}{2} E \lambda w_{, x}^{2}-f(x) w\right) d x,
$$

where $\mathcal{U}_{a}$ is the kinematically admissible space, in which certain necessary boundary conditions are given. Thus, for the given external load $f(x)$ and end load $\lambda$, the primal variational problem is to find $\bar{w} \in \mathcal{U}_{a}$ such that

$$
(\mathcal{P}): \Pi(\bar{w})=\inf \left\{\Pi(w) \mid w \in \mathcal{U}_{a}\right\} .
$$

It is easy to prove that the stationary condition $\delta \Pi(w)=0$ leads to the governing equation (2.1).

If the nonlinear term in (2.1) is ignored and $f=0$, then this nonlinear Gao beam is degeneralized to the well-known Euler-Bernoulli beam equation ${ }^{1}$ :

$$
E I w_{, x x x x}+\lambda E w_{, x x}=0 .
$$

It is known that this linear beam will be buckled if the axial load $\lambda$ reaches the Euler buckling load $\lambda_{c r}$ defined by

$$
\lambda_{c r}=\inf _{w \in \mathcal{U}_{a}} \frac{\int_{0}^{L} E I w_{, x x}^{2} d x}{\int_{0}^{L} E w_{, x}^{2} d x} .
$$

Clearly, in the pre-buckling state, i.e. before the axial load $\lambda$ reaches the Euler buckling load $\lambda_{c r}$, we have

$$
\Pi_{E B}(w)=\int_{0}^{L} E I w_{, x x}^{2} d x-\lambda \int_{0}^{L} E w_{, x}^{2} d x>0 \forall w \in \mathcal{U}_{a}, \lambda<\lambda_{c r} .
$$

\footnotetext{
${ }^{1}$ Strictly speaking, instead of $\lambda$, the axial load in the Euler-Bernoulli beam should be $F=\lambda E /[(1+$ $\left.\mu)\left(1-\mu^{2}\right)\right]$.
} 
In this case, $\Pi_{E B}(w)$ and $\Pi(w)$ are strictly convex on $\mathcal{U}_{a}$, therefore, both the EulerBernoulli beam (2.5) and the nonlinear Gao beam (2.1) can have only one solution (see Lemma 2.1. and Theorem 2.1 in [27]).

Dually, in the post-buckling state, i.e. $\lambda>\lambda_{c r}$, the total potential energy for the Euler-Bernoulli beam is strictly concave and

$$
\inf \left\{\Pi_{E B}(w) \mid w \in \mathcal{U}_{a}, \lambda>\lambda_{c r}\right\}=-\infty,
$$

which means that the Euler-Bernoulli beam is crushed. This shows that the EulerBernoulli beam can't be used for studying post-buckling problems. However, for the nonlinear Gao beam, it was proved recently by Machalová and Netuka (see Remark 2.2, [27]) that there exists a constant $\lambda_{c r}^{G} \geq \lambda_{c r}$ such that the total potential energy $\Pi(w)$ is a nonconvex (double-well) functional if $\lambda>\lambda_{c r}^{G}$, which allows at most three critical points, i.e. the strong solutions to the nonlinear equation $(2.1)$ at each material point $x \in[0, L]$ : two minimizers corresponding to the two possible buckled states, one local maximizer corresponding to the possible unbuckled state [14]. Clearly, these solutions are sensitive to both the axial load $\lambda$ and the distributed lateral force field $f(x)$. By equation (2.2) we know that the axial deformation could be relatively large, while the nonconvexity of the total potential shows that this nonlinear beam model can be used for studying both pre and post-buckling problems $[3,30]$. Recently, the Gao beam model has been generalized for many real-world applications in engineering and sciences $[1,2,21,22,24-26,28]$.

Although the nonlinear Gao beam can be used for modeling natural phenomena, the nonconvexity of this beam model leads to some fundamental challenges in mathematics and computational science. Generally speaking, traditional numerical methods and nonlinear optimization techniques can be used only for solving convex minimization problems. Due to the lack of a global optimality criterion to identify a global minimizer at each iteration, most nonconvex optimization problems can't be solved deterministically, therefore, they are considered to be NP-hard in global optimization and computer science [17].

It was shown in [10] that by introducing a canonical strain measure $\epsilon=\Lambda(w)=\frac{1}{2} w_{, x}^{2}$ and a convex canonical function $V(\epsilon)=\frac{1}{3} E \alpha \epsilon^{2}-E \lambda \epsilon$, the nonconvex (double-well) potential $W\left(w_{, x}\right)=\frac{1}{12} E \alpha w_{, x}^{4}-\frac{1}{2} E \lambda w_{, x}^{2}$ in $\Pi$ can be written in the canonical form $W\left(w_{, x}\right)=V(\Lambda(w))$. Thus, the canonical dual stress can be uniquely defined by

$$
\sigma=\partial V(\epsilon)=\frac{2 E \alpha}{3} \epsilon-E \lambda
$$

By the Legendre transformation, we have the canonical complementary energy

$$
V^{*}(\sigma)=\epsilon \sigma-V(\epsilon)=\frac{3}{4 E \alpha}(\sigma+E \lambda)^{2} .
$$

Thus, replacing $W\left(w_{, x}\right)$ with $V(\Lambda(w))=\Lambda(w) \sigma-V^{*}(\sigma)$, the Gao-Strang total complementary energy $\Xi: \mathcal{U}_{a} \times \mathcal{S}_{a} \rightarrow \mathbb{R}[18]$ in nonlinear elasticity can be defined as

$$
\begin{aligned}
\Xi(w, \sigma) & =\int_{0}^{L}\left(\frac{1}{2} E I w_{, x x}^{2}+\frac{1}{2} \sigma w_{, x}^{2}-\frac{3}{4 E \alpha}(\sigma+E \lambda)^{2}-f(x) w\right) d x \\
& =G(w, \sigma)-\int_{0}^{L}\left[V^{*}(\sigma)-f(x) w\right] d x,
\end{aligned}
$$

where $\mathcal{S}_{a}=\{\sigma \in C[0, L] \mid \sigma(x) \geq-\lambda E \forall x \in[0, L]\}$ and

$$
G(w, \sigma)=\int_{0}^{L}\left(\frac{1}{2} E I w_{, x x}^{2}+\frac{1}{2} \sigma w_{, x}^{2}\right) d x
$$


is the generalized Gao-Strang complementary gap function [18].

TheOrem 2.1 (Complementary-duality Principle). For any given external load $f(x)$ and end load $\lambda$, the pair $(\bar{w}, \bar{\sigma})$ is a critical point of $\Xi(w, \sigma)$ if and only if $\bar{w}$ is a critical point of $\Pi(w)$ and $\Pi(\bar{w})=\Xi(\bar{w}, \bar{\sigma})$.

Proof. The criticality condition $\delta \Xi(\bar{w}, \bar{\sigma})=0$ leads to the following canonical equations:

$$
\begin{aligned}
& E I \bar{w}_{, x x x x}-\bar{\sigma} \bar{w}_{, x x}=f(x), \\
& \frac{1}{2} \bar{w}_{, x}^{2}=\frac{3}{2 E \alpha}(\bar{\sigma}+E \lambda),
\end{aligned}
$$

which are equivalent to Equation (2.1). The equality $\Pi(\bar{w})=\Xi(\bar{w}, \bar{\sigma})$ follows directly from the Fenchel-Young equality $V(\Lambda(\bar{w}))+V^{*}(\bar{\sigma})=\Lambda(\bar{w}) \bar{\sigma}$ due to the convexity of the canonical function $V(\epsilon)$.

TheOREm 2.2 (Triality Theory). Let $(\bar{w}, \bar{\sigma})$ be a critical point of $\Xi(w, \sigma)$.

If $G(\bar{w}, \bar{\sigma}) \geq 0$, then $\bar{w}$ is a global minimizer of $\Pi(w)$ on $\mathcal{U}_{a}$ and

$$
\Pi(\bar{w})=\min _{w \in \mathcal{U}_{a}} \Pi(w)=\min _{w \in \mathcal{U}_{a}} \max _{\sigma \in \mathcal{S}_{a}} \Xi(w, \sigma) .
$$

If $G(\bar{w}, \bar{\sigma})<0$, then on a neighborhood $\mathcal{U}_{o} \times \mathcal{S}_{o}$ of $(\bar{w}, \bar{\sigma})$, we have either

$$
\Pi(\bar{w})=\min _{w \in \mathcal{U}_{o}} \Pi(w)=\min _{w \in \mathcal{U}_{o}} \max _{\sigma \in \mathcal{S}_{o}} \Xi(w, \sigma)=\Xi(\bar{w}, \bar{\sigma}),
$$

or

$$
\Pi(\bar{w})=\max _{w \in \mathcal{U}_{o}} \Pi(w)=\max _{w \in \mathcal{U}_{o}} \max _{\sigma \in \mathcal{S}_{o}} \Xi(w, \sigma)=\Xi(\bar{w}, \bar{\sigma}) .
$$

Proof. For the positive gap function, $\Xi(w, \sigma)$ is a saddle functional and the total potential $\Pi(w)$ is convex on $\mathcal{U}_{a}$ [18]. In this case, statement (2.14) follows directly from Gao and Strang's theory for general large deformation problems [18]. While for the negative gap function, $\Xi(w, \sigma)$ is a bi-concave functional. In this case, the total potential $\Pi(w)$ is nonconvex on $\mathcal{U}_{a}$, which could have both local minimum and local maximum solutions. Due to the fact that $\max _{\sigma \in \mathcal{S}_{o}} \Xi(w, \sigma)=\max _{w \in \mathcal{U}_{o}} \Pi(w)$, the statements $(2.15)$ and (2.16) can be proved easily by the general triality theory [10].

The triality theory was first discovered in the post-buckling analysis of the large deformed beam theory [8]. Generalization to nonconvex/discrete optimization problems was given in 2000 [11]. Detailed information relating to this theory as well as its extensive applications in global optimization as well as nonconvex mechanics can be found in the monograph [10] and recent review articles $[11,15,16]$.

\section{Mixed finite element method}

By using the finite element method, the domain of the beam is discretized into $m$ elements $[0, L]=\bigcup_{e=1}^{m} \Omega^{e}$. In each element $\Omega^{e}=\left[x^{e}, x^{e+1}\right]$, the deflection, rotating angular and dual stress for node $x^{e}$ are marked as $w^{e}, \theta^{e}$ and $\sigma^{e}$, respectively, and similar for node $x^{e+1}$. Then, we have the nodal displacement vector $w_{e}$ of the e-th element

$$
w_{e}^{T}=\left[w^{e} \theta^{e} w^{e+1} \theta^{e+1}\right]
$$


and the nodal dual stress element $\sigma_{e}$

$$
\sigma_{e}^{T}=\left[\sigma^{e} \sigma^{e+1}\right]
$$

In each element, we use mixed finite element interpolations for both $w(x)$ and $\sigma(x)$, i.e.

$$
w_{e}^{h}(x)=N_{w}^{T}(x) w_{e}, \quad \sigma_{e}^{h}(x)=N_{\sigma}^{T}(x) \sigma_{e} \forall x \in \Omega^{e} .
$$

Thus, the spaces $\mathcal{U}_{a}$ and $\mathcal{S}_{a}$ can be numerically discretized to the finite-dimensional spaces $\mathcal{U}_{a}^{h}$ and $\mathcal{S}_{a}^{h}$, respectively. In this paper, the shape functions are based on piecewise-cubic polynomial for $w(x)$ and piecewise-linear for $\sigma(x)$, i.e.

$$
N_{w}=\left[\begin{array}{c}
\frac{1}{4}(1-\xi)^{2}(2+\xi) \\
\frac{L_{e}}{8}(1-\xi)^{2}(1+\xi) \\
\frac{1}{4}(1+\xi)^{2}(2-\xi) \\
\frac{L_{e}}{8}(1+\xi)^{2}(\xi-1)
\end{array}\right], \quad N_{\sigma}=\frac{1}{2}\left[\begin{array}{c}
(1-\xi) \\
(1+\xi)
\end{array}\right],
$$

where $\xi=2 x / L_{e}-1$ with $L_{e}$ is the length of the e-th beam element. Thus, on the discretized feasible deformation space $\mathcal{U}_{a}^{h}$, the Gao-Strang total complementary energy can be expressed in the following discretized form

$$
\begin{aligned}
\Xi^{h}(\mathbf{w}, \boldsymbol{\sigma}) & =\sum_{e=1}^{m}\left(\frac{1}{2} w_{e}^{T} G^{e}\left(\sigma_{e}\right) w_{e}-\frac{1}{2} \sigma_{e}^{T} K_{e} \sigma_{e}-\lambda_{e}^{T} \sigma_{e}-f_{e}^{T} w_{e}-c_{e}\right) \\
& =\frac{1}{2} \mathbf{w}^{T} \mathbf{G}(\boldsymbol{\sigma}) \mathbf{w}-\frac{1}{2} \boldsymbol{\sigma}^{T} \mathbf{K} \boldsymbol{\sigma}-\boldsymbol{\lambda}^{T} \boldsymbol{\sigma}-\mathbf{f}^{T} \mathbf{w}-c,
\end{aligned}
$$

where $\mathbf{w} \in \mathcal{U}_{a}^{h} \subset \mathbb{R}^{2(m+1)}$ and $\boldsymbol{\sigma} \in \mathcal{S}_{a}^{h} \subset \mathbb{R}^{m+1}$ are nodal deflection and dual stress vectors, respectively. We let

$$
\mathcal{S}_{a}^{h}=\left\{\boldsymbol{\sigma} \in \mathbb{R}^{m+1} \mid \operatorname{det} \mathbf{G}(\boldsymbol{\sigma}) \neq 0\right\} .
$$

The Hessian matrix of the gap function $\mathbf{G}(\boldsymbol{\sigma}) \in \mathbb{R}^{2(m+1)} \times \mathbb{R}^{2(m+1)}$ is obtained by assembling the following symmetric matrices $G^{e}\left(\sigma_{e}\right)$ :

$$
\begin{aligned}
G^{e}\left(\sigma_{e}\right) & =\int_{\Omega_{e}}\left(E I N_{w}^{\prime \prime}\left(N_{w}^{\prime \prime}\right)^{T}+\left(N_{\sigma}\right)^{T} \sigma_{e} N_{w}^{\prime}\left(N_{w}^{\prime}\right)^{T}\right) d x \\
& =\int_{-1}^{1} \frac{L_{e}}{2}\left(E I N_{w}^{\prime \prime}\left(N_{w}^{\prime \prime}\right)^{T}+\left(N_{\sigma}\right)^{T} \sigma_{e} N_{w}^{\prime}\left(N_{w}^{\prime}\right)^{T}\right) d \xi=\left[G_{i j}^{e}\right]_{4 \times 4}
\end{aligned}
$$

where $G_{i j}^{e}=G_{j i}^{e}$ are defined by the two stress ends $\sigma^{e}$ and $\sigma^{e+1}$ of beam element as:

$$
G^{e}=\left[\begin{array}{cccc}
\frac{3\left(\sigma^{e}+\sigma^{e+1}\right)}{5 L_{e}}+\frac{12 E I}{L_{e}^{3}} & \frac{\sigma^{e+1}}{10}+\frac{6 E I}{L_{e}^{2}} & -G_{11}^{e} & \frac{\sigma^{e}}{10}+\frac{6 E I}{L_{e}^{2}} \\
G_{12}^{e} & L_{e}\left(\frac{\sigma^{e}}{10}+\frac{\sigma^{e+1}}{30}\right)+\frac{4 E I}{L_{e}} & -G_{12}^{e} & -\frac{L_{e}}{60}\left(\sigma^{e}+\sigma^{e+1}\right)+\frac{2 E I}{L_{e}} \\
-G_{11}^{e} & G_{23}^{e} & G_{11}^{e} & -G_{14}^{e} \\
G_{14}^{e} & G_{24}^{e} & G_{34}^{e} & L_{e}\left(\frac{\sigma^{e}}{30}+\frac{\sigma^{e+1}}{10}\right)+\frac{4 E I}{L_{e}}
\end{array}\right] .
$$

The matrix $\mathbf{K} \in \mathbb{R}^{m+1} \times \mathbb{R}^{m+1}$ is obtained by assembling the following positivedefinite matrices $K_{e}$

$$
K_{e}=\int_{\Omega_{e}}\left(\frac{3}{2 E \alpha} N_{\sigma} N_{\sigma}^{T}\right) d x=\int_{-1}^{1}\left(\frac{3 L_{e}}{4 E \alpha} N_{\sigma} N_{\sigma}^{T}\right) d \xi=\frac{L_{e}}{E \alpha}\left[\begin{array}{cc}
\frac{1}{2} & \frac{1}{4} \\
\frac{1}{4} & \frac{1}{2}
\end{array}\right] .
$$


Also, $\boldsymbol{\lambda}=\left\{\lambda_{e}\right\} \in \mathbb{R}^{m+1}$ and $\mathbf{f}=\left\{f_{e}\right\} \in \mathbb{R}^{2(m+1)}$ are defined by assembling the following

$$
\begin{gathered}
\lambda_{e}=\int_{\Omega_{e}}\left(\frac{3}{2 \alpha} \lambda N_{\sigma}\right) d x=\int_{-1}^{1}\left(\frac{3 L_{e}}{4 \alpha} \lambda N_{\sigma}\right) d \xi=\frac{\lambda L_{e}}{\alpha}\left[\begin{array}{c}
\frac{3}{4} \\
\frac{3}{4}
\end{array}\right], \\
f_{e}=\int_{\Omega_{e}} f(x) N_{w} d x=\int_{-1}^{1} \frac{L_{e}}{2} f(\xi) N_{w} d \xi
\end{gathered}
$$

and $c=\sum_{e=1}^{m} c_{e} \in \mathbb{R}$ is defined as

$$
c_{e}=\int_{\Omega_{e}}\left(\frac{3 E}{4 \alpha} \lambda^{2}\right) d x=\int_{-1}^{1}\left(\frac{3 E L_{e}}{8 \alpha} \lambda^{2}\right) d \xi=\frac{3}{4 \alpha} E L_{e} \lambda^{2} .
$$

By the critical condition $\delta \Xi^{h}(\mathbf{w}, \boldsymbol{\sigma})=0$, canonical Equations (2.12) and (2.13) have the following discretized forms

$$
\begin{gathered}
\mathbf{G}(\boldsymbol{\sigma}) \mathbf{w}-\mathbf{f}=0, \\
\frac{1}{2} \mathbf{w}^{T} \mathbf{H} \mathbf{w}-\mathbf{K} \boldsymbol{\sigma}-\boldsymbol{\lambda}=0,
\end{gathered}
$$

where $\mathbf{H}=\mathbf{G}, \boldsymbol{\sigma}(\boldsymbol{\sigma})$ stands for gradient of $\mathbf{G}(\boldsymbol{\sigma})$ with respect to the vector $\boldsymbol{\sigma}$ and

$$
\mathbf{w}^{T} \mathbf{H w}=\left[\begin{array}{c}
\mathbf{w}^{T} \mathbf{G}_{,} \boldsymbol{\sigma}_{1}(\boldsymbol{\sigma}) \mathbf{w} \\
\mathbf{w}^{T} \mathbf{G}_{,} \boldsymbol{\sigma}_{2}(\boldsymbol{\sigma}) \mathbf{w} \\
\cdot \\
\cdot \\
\cdot \\
\mathbf{w}^{T} \mathbf{G}_{,} \boldsymbol{\sigma}_{m+1}(\boldsymbol{\sigma}) \mathbf{w}
\end{array}\right] \in \mathbb{R}^{m+1}
$$

in which $\boldsymbol{\sigma}^{T}=\left[\boldsymbol{\sigma}_{1}, \boldsymbol{\sigma}_{2}, \ldots, \boldsymbol{\sigma}_{m+1}\right]$.

For any given $\mathbf{w} \in \mathcal{U}_{a}^{h}$, we know that $\Xi(\mathbf{w}, *): \mathcal{S}_{a}^{h} \rightarrow \mathbb{R}$ is concave and the discretized total potential energy can be obtained by

$$
\Pi_{p}^{h}(\mathbf{w})=\max \left\{\Xi(\mathbf{w}, \boldsymbol{\sigma}) \mid \boldsymbol{\sigma} \in \mathcal{S}_{a}^{h}\right\}=\left\{\Xi(\mathbf{w}, \boldsymbol{\sigma}) \mid \boldsymbol{\sigma}=\mathbf{K}^{-1}\left(\frac{1}{2} \mathbf{w}^{T} \mathbf{H} \mathbf{w}-\boldsymbol{\lambda}\right)\right\} .
$$

However, the convexity $\Xi(*, \boldsymbol{\sigma}): \mathcal{U}_{a}^{h} \rightarrow \mathbb{R}$ will depend on $\boldsymbol{\sigma} \in \mathcal{S}_{a}^{h}$. The discretized pure complementary energy $\Pi_{d}^{h}: \mathcal{S}_{a}^{h} \rightarrow \mathbb{R}$ can be obtained by the following canonical dual transformation

$$
\begin{aligned}
\Pi_{d}^{h}(\boldsymbol{\sigma}) & =\operatorname{sta}\left\{\Xi(\mathbf{w}, \boldsymbol{\sigma}) \mid \mathbf{w} \in \mathcal{U}_{a}^{h}\right\}=\left\{\Xi(\mathbf{w}, \boldsymbol{\sigma}) \mid \mathbf{w}=\mathbf{G}^{-1}(\boldsymbol{\sigma}) \mathbf{f}\right\} \\
& =-\frac{1}{2} \mathbf{f}^{T} \mathbf{G}^{-1}(\boldsymbol{\sigma}) \mathbf{f}-\frac{1}{2} \boldsymbol{\sigma}^{T} \mathbf{K} \boldsymbol{\sigma}-\boldsymbol{\lambda}^{T} \boldsymbol{\sigma}-c
\end{aligned}
$$

where sta $\left\{g(\mathbf{w}) \mid \mathbf{w} \in \mathcal{U}_{a}^{h}\right\}$ stands for finding the stationary value of $g(\mathbf{w})$ on $\mathcal{U}_{a}^{h}$. Clearly, its convexity depends on $\mathbf{G}(\boldsymbol{\sigma})$. Let

$$
\begin{aligned}
& \mathcal{S}_{a}^{+}=\left\{\boldsymbol{\sigma} \in \mathcal{S}_{a}^{h} \mid \mathbf{G}(\boldsymbol{\sigma}) \succ 0\right\}, \\
& \mathcal{S}_{a}^{-}=\left\{\boldsymbol{\sigma} \in \mathcal{S}_{a}^{h} \mid \mathbf{G}(\boldsymbol{\sigma}) \prec 0\right\} .
\end{aligned}
$$


Where the symbols " $\succ$ " and " $\prec$ " represent the positive definite matrix and negative definite matrix, respectively.

THEOREM 3.1. Suppose $(\overline{\mathbf{w}}, \overline{\boldsymbol{\sigma}})$ is a stationary point of $\Xi^{h}(\mathbf{w}, \boldsymbol{\sigma})$, then $\Pi_{p}^{h}(\overline{\mathbf{w}})=$ $\Xi^{h}(\overline{\mathbf{w}}, \overline{\boldsymbol{\sigma}})=\Pi_{d}^{h}(\overline{\boldsymbol{\sigma}})$. Moreover, if $\overline{\boldsymbol{\sigma}} \in \mathcal{S}_{a}^{+}$, then we have

Canonical Min-Max Duality: $\overline{\mathbf{w}}$ is a global minimizer of $\Pi_{p}^{h}(\mathbf{w})$ on $\mathcal{U}_{a}^{h}$ if and only if $\overline{\boldsymbol{\sigma}}$ is a global maximizer of $\Pi_{d}^{h}(\boldsymbol{\sigma})$ on $\mathcal{S}_{a}^{+}$, i.e.,

$$
\Pi_{p}^{h}(\overline{\mathbf{w}})=\min _{\mathbf{w} \in \mathcal{U}_{a}^{h}} \Pi_{p}^{h}(\mathbf{w}) \Leftrightarrow \max _{\boldsymbol{\sigma} \in \mathcal{S}_{a}^{+}} \Pi_{d}^{h}(\boldsymbol{\sigma})=\Pi_{d}^{h}(\overline{\boldsymbol{\sigma}}) .
$$

If $\overline{\boldsymbol{\sigma}} \in \mathcal{S}_{a}^{-}$, then on a neighborhood $\mathcal{U}_{o} \times \mathcal{S}_{o} \subset \mathcal{U}_{a}^{h} \times \mathcal{S}_{a}^{-}$of $(\overline{\mathbf{w}}, \overline{\boldsymbol{\sigma}})$ we have

Canonical Double-max Duality: The stationary point $\overline{\mathbf{w}}$ is a local maximizer of $\Pi_{p}^{h}(\mathbf{w})$ on $\mathcal{U}_{o}$ if and only if the stationary point $\overline{\boldsymbol{\sigma}}$ is a local maximizer of $\Pi_{d}^{h}(\boldsymbol{\sigma})$ on $\mathcal{S}_{\text {o }}$, i.e.,

$$
\Pi_{p}^{h}(\overline{\mathbf{w}})=\max _{\mathbf{w} \in \mathcal{U}_{o}} \Pi_{p}^{h}(\mathbf{w}) \Leftrightarrow \max _{\boldsymbol{\sigma} \in \mathcal{S}_{o}} \Pi_{d}^{h}(\boldsymbol{\sigma})=\Pi_{d}^{h}(\overline{\boldsymbol{\sigma}})
$$

Canonical Double-min Duality (if $\operatorname{dim} \mathcal{U}_{a}^{h}=\operatorname{dim} \mathcal{S}_{a}^{h}$ ): The stationary point $\overline{\mathbf{w}}$ is a local minimizer of $\Pi_{p}^{h}(\mathbf{w})$ on $\mathcal{U}_{o}$ if and only if the stationary point $\overline{\boldsymbol{\sigma}}$ is a local minimizer of $\Pi_{d}^{h}(\boldsymbol{\sigma})$ on $\mathcal{S}_{o}$, i.e.,

$$
\Pi_{p}^{h}(\overline{\mathbf{w}})=\min _{\mathbf{w} \in \mathcal{U}_{o}} \Pi_{p}^{h}(\mathbf{w}) \Leftrightarrow \min _{\boldsymbol{\sigma} \in \mathcal{S}_{o}} \Pi_{d}^{h}(\boldsymbol{\sigma})=\Pi_{d}^{h}(\overline{\boldsymbol{\sigma}})
$$

The proof of this theorem follows from the general results in global optimization $[4,19,29]$. Canonical min-max duality can be used to find the global minimizer of the nonconvex problem via the following canonical dual problem:

$$
\left(\mathcal{P}^{d}\right): \max \left\{\Pi_{d}^{h}(\boldsymbol{\sigma}) \mid \boldsymbol{\sigma} \in \mathcal{S}_{a}^{+}\right\},
$$

which is a concave maximization problem and can be solved easily by well-developed convex analysis and optimization techniques. The canonical double-max and doublemin duality statements can be used to find the biggest local maximizer and a local minimizer of the nonconvex primal problem, respectively. It was proved in $[4,19,29]$ that both canonical min-max and double-max duality statements hold strongly regardless of the dimensions of $\mathcal{U}_{a}^{h}$ and $\mathcal{S}_{a}^{h}$, while the canonical double-min duality statement (3.14) holds strongly for $\operatorname{dim} \mathcal{U}_{a}^{h}=\operatorname{dim} \mathcal{S}_{a}^{h}$, but weakly if $\operatorname{dim} \mathcal{U}_{a}^{h} \neq \operatorname{dim} \mathcal{S}_{a}^{h}$. This case is within our reach in the following applications.

\section{Semi-Definite Programming algorithm}

It is easy to understand that the nonconvex post-buckling problem could have multiple global minimizers for certain external loads, say $q(x)=0$. In this case we have $\operatorname{det} \mathbf{G}(\boldsymbol{\sigma})=0$ and $\mathcal{S}_{a}^{h}=\emptyset$. In order to deal with this case, this section presents a SDP (Semi-Definite Programming, see [31] and [32]) reformulation to solve the canonical dual problem (3.15). The SDP algorithm is applied to obtain all post-buckled solutions of a large deformed elastic beam.

By the fact that $\Xi(\mathbf{w}, \boldsymbol{\sigma})$ is a saddle function on $\mathcal{U}_{a}^{h} \times \mathcal{S}_{a}^{+}$, we have

$$
\min _{\mathbf{w} \in \mathcal{U}_{a}^{h}} \Pi_{p}^{h}(\mathbf{w})=\min _{\mathbf{w} \in \mathcal{U}_{a}^{h}} \max _{\boldsymbol{\sigma} \in \mathcal{S}_{a}^{+}} \Xi(\mathbf{w}, \boldsymbol{\sigma})=\max _{\boldsymbol{\sigma} \in \mathcal{S}_{a}^{+}} \min _{\mathbf{w} \in \mathcal{U}_{a}^{h}} \Xi(\mathbf{w}, \boldsymbol{\sigma}) .
$$

For any given $\boldsymbol{\sigma} \in \mathcal{S}_{a}^{+}$, the solution to $\min _{\mathbf{w} \in \mathcal{U}_{a}^{h}} \Xi(\mathbf{w}, \boldsymbol{\sigma})$ leads to

$$
\mathbf{w}=\mathbf{w}(\boldsymbol{\sigma})=\mathbf{G}^{-1}(\boldsymbol{\sigma}) \mathbf{f}
$$


Thus, the stress fields $\boldsymbol{\sigma}$ can be found by the following problem

$$
\begin{aligned}
\max _{\boldsymbol{\sigma}} \Xi(\mathbf{w}(\boldsymbol{\sigma}), \boldsymbol{\sigma}) & =\frac{1}{2} \mathbf{w}(\boldsymbol{\sigma})^{T} \mathbf{G}(\boldsymbol{\sigma}) \mathbf{w}(\boldsymbol{\sigma})-\frac{1}{2} \boldsymbol{\sigma}^{T} \mathbf{K} \boldsymbol{\sigma}-\boldsymbol{\lambda}^{T} \boldsymbol{\sigma}-\mathbf{f}^{T} \mathbf{w}(\boldsymbol{\sigma})-c \\
& \equiv \max _{\boldsymbol{\sigma}} \Pi_{d}^{h}(\boldsymbol{\sigma}) \\
\text { s.t. } \quad \mathbf{G}(\boldsymbol{\sigma}) & \succeq 0,
\end{aligned}
$$

where the symbol " $\succeq$ " represents the positive semi-definite matrix. By canonical min$\max$ duality we know that if $\boldsymbol{\sigma}^{*} \in \mathcal{S}_{a}^{+}$is a global maximizer of problem (4.3), then $\mathbf{w}^{*}=\mathbf{w}\left(\boldsymbol{\sigma}^{*}\right)$ should be a global minimizer of $\Pi_{p}^{h}(\mathbf{w})$. Furthermore, the problem (4.3) is the same as:

$$
\max _{\boldsymbol{\sigma}, t} t \text { s.t. } \mathbf{G}(\boldsymbol{\sigma}) \succeq 0, \quad t \leq \phi(\boldsymbol{\sigma})-\frac{1}{2} \boldsymbol{\sigma}^{T} \mathbf{K} \boldsymbol{\sigma}
$$

where $\phi(\boldsymbol{\sigma})=\frac{1}{2} \mathbf{w}(\boldsymbol{\sigma})^{T} \mathbf{G}(\boldsymbol{\sigma}) \mathbf{w}(\boldsymbol{\sigma})-\boldsymbol{\lambda}^{T} \boldsymbol{\sigma}-\mathbf{f}^{T} \mathbf{w}(\boldsymbol{\sigma})-c$. By the fact that $\mathbf{K} \succ 0$, the Schur complement lemma (see [32]) for the second inequality constraint in (4.4) implies

$$
\left[\begin{array}{cc}
2 \mathbf{K}^{-1} & \boldsymbol{\sigma} \\
\boldsymbol{\sigma}^{T} & \phi(\boldsymbol{\sigma})-t
\end{array}\right] \succeq 0
$$

Thus, the problem (4.4) can be relaxed to the following Semi-Definite Programming (SDP) problem

$$
\max _{\boldsymbol{\sigma}, t} t \text { s.t. } \mathbf{G}(\boldsymbol{\sigma}) \succeq 0,\left[\begin{array}{cc}
2 \mathbf{K}^{-1} & \boldsymbol{\sigma} \\
\boldsymbol{\sigma}^{T} & \phi(\boldsymbol{\sigma})-t
\end{array}\right] \succeq 0 .
$$

In the same way, the SDP relaxation for the canonical double-max duality statement

$$
\max _{\mathbf{w} \in \mathcal{U}_{a}^{h}} \Pi_{p}^{h}(\mathbf{w})=\max _{\mathbf{w}, \boldsymbol{\sigma}} \Xi(\mathbf{w}, \boldsymbol{\sigma})=\max \Pi_{d}^{h}(\boldsymbol{\sigma}) \text { s.t. } \quad \boldsymbol{\sigma} \in \mathcal{S}_{a}^{-}
$$

should be equivalent to

$$
\max _{\boldsymbol{\sigma}, t} t \text { s.t. } \quad-\mathbf{G}(\boldsymbol{\sigma}) \succ 0,\left[\begin{array}{cc}
2 \mathbf{K}^{-1} & \boldsymbol{\sigma} \\
\boldsymbol{\sigma}^{T} & \phi(\boldsymbol{\sigma})-t
\end{array}\right] \succeq 0,
$$

which leads to a local maximum solution to the post-buckling problem.

Now, let $\left(\mathbf{w}^{*}, \boldsymbol{\sigma}^{*}\right)$ be a local minimizer of the canonical double-min problem $\min _{\mathbf{w}} \Pi_{p}^{h}(\mathbf{w})=\min _{\mathbf{w}} \max \boldsymbol{\sigma} \Xi(\mathbf{w}, \boldsymbol{\sigma})=\min _{\boldsymbol{\sigma}} \Pi_{d}^{h}(\boldsymbol{\sigma})$ s.t. $\boldsymbol{\sigma} \in \mathcal{S}_{a}^{-}$. By Equation (4.2), the local minimizer is equivalent to the following problem

$$
\min _{\boldsymbol{\sigma}}\left\{\Xi(\mathbf{w}(\boldsymbol{\sigma}), \boldsymbol{\sigma}) \equiv \Pi_{d}^{h}(\boldsymbol{\sigma})\right\} \quad \text { s.t. } \mathbf{G}(\boldsymbol{\sigma}) \prec 0 .
$$

This problem is the same as:

$$
\min _{\boldsymbol{\sigma}, t} t \text { s.t. } \mathbf{G}(\boldsymbol{\sigma}) \prec 0, t \geq-\frac{1}{2} \mathbf{f}^{T} \mathbf{G}^{-1}(\boldsymbol{\sigma}) \mathbf{f}-\frac{1}{2} \boldsymbol{\sigma}^{T} \mathbf{K} \boldsymbol{\sigma}-\boldsymbol{\lambda}^{T} \boldsymbol{\sigma}-c .
$$

In order to apply the Schur complement lemma for the second inequality in (4.10), we need to linearize the complementary energy $V^{*}(\boldsymbol{\sigma})=\frac{1}{2} \boldsymbol{\sigma}^{T} \mathbf{K} \boldsymbol{\sigma}$. This can be done by using a reformulated pure complementary energy:

$$
\widehat{\Pi}^{d}(\boldsymbol{\sigma}, \mathbf{w})=-\frac{1}{2} \mathbf{f}^{T} \mathbf{G}^{-1}(\boldsymbol{\sigma}) \mathbf{f}-\frac{1}{2} \mathbf{w}^{T} \mathbf{M}(\boldsymbol{\sigma}) \mathbf{w}-\frac{1}{2} \boldsymbol{\lambda}^{T} \boldsymbol{\sigma}-c,
$$


the stiffness matrix $\mathbf{M}(\boldsymbol{\sigma})$ in the strain energy $V(\mathbf{w})=\frac{1}{2} \mathbf{w}^{T} \mathbf{M}(\boldsymbol{\sigma}) \mathbf{w}=V^{*}(\boldsymbol{\sigma})$ is obtained by assembling the following symmetric matrices $M^{e}\left(\sigma_{e}\right)$ in each element

$$
\begin{aligned}
M^{e}\left(\sigma_{e}\right)= & \int_{\Omega_{e}} \frac{1}{2}\left(\left(N_{\sigma}\right)^{T} \sigma_{e} N_{w}^{\prime}\left(N_{w}^{\prime}\right)^{T}\right) d x=\int_{-1}^{1} \frac{L_{e}}{4}\left(\left(N_{\sigma}\right)^{T} \sigma_{e} N_{w}^{\prime}\left(N_{w}^{\prime}\right)^{T}\right) d \xi \\
= & {\left[\begin{array}{cccc}
\frac{3}{10 L_{e}}\left(\sigma^{e}+\sigma^{e+1}\right) & \frac{1}{20} \sigma^{e+1} & -M_{1,1}^{e} & \frac{1}{20} \sigma^{e} \\
M_{12}^{e} & \frac{L_{e}}{60}\left(3 \sigma^{e}+\sigma^{e+1}\right) & -M_{12}^{e} & \frac{-L_{e}}{120}\left(\sigma^{e}+\sigma^{e+1}\right) \\
M_{13}^{e} & M_{23}^{e} & M_{11}^{e} & -M_{14}^{e} \\
M_{14}^{e} & M_{24}^{e} & M_{34}^{e} & \frac{L_{e}}{60}\left(\sigma^{e}+3 \sigma^{e+1}\right)
\end{array}\right] . }
\end{aligned}
$$

Therefore, by using $\widehat{\Pi}^{d}(\boldsymbol{\sigma}, \mathbf{w})$, problem (4.10) can be relaxed to

$$
\min _{\boldsymbol{\sigma}, t} t \text { s.t. } \mathbf{G}(\boldsymbol{\sigma}) \prec 0, \frac{1}{2} \mathbf{f}^{T} \mathbf{G}^{-1}(\boldsymbol{\sigma}) \mathbf{f}+\hat{\phi}(\boldsymbol{\sigma}, \mathbf{w})+t \geq 0,
$$

where $\hat{\phi}(\boldsymbol{\sigma}, \mathbf{w})=\frac{1}{2} \mathbf{w}^{T} \mathbf{M}(\boldsymbol{\sigma}) \mathbf{w}+\frac{1}{2} \boldsymbol{\lambda}^{T} \boldsymbol{\sigma}+c$. The primal variable $\mathbf{w}$ in this problem can be computed by the dual solution $\sigma$ in the primal-dual iteration. Thus, by using the Schur complement lemma this problem can be relaxed to the following SDP problem

$$
\min _{\boldsymbol{\sigma}, t} t \text { s.t. } \quad-\mathbf{G}(\boldsymbol{\sigma}) \succ 0,\left[\begin{array}{cc}
-2 \mathbf{G}(\boldsymbol{\sigma}) & \mathbf{f} \\
\mathbf{f}^{T} & \hat{\phi}(\boldsymbol{\sigma}, \mathbf{w})+t
\end{array}\right] \succeq 0,
$$

Clearly, if stress $\boldsymbol{\sigma}^{*}$ is a local minimizer on $\mathcal{S}_{a}^{-}$of problem (4.14), the canonical doublemin duality shows that $\mathbf{w}^{*}=\mathbf{w}\left(\boldsymbol{\sigma}^{*}\right)$ should be a local minimizer of $\Pi_{p}^{h}(\mathbf{w})$.

Consequently, the primal-dual semi-definite programming (PD-SDP) algorithm for solving all possible post-buckling solutions can be proposed as the following.

\section{PD-SDP Algorithm:}

(1) Given initial primal solution $\mathbf{w}^{(0)}$ and error allowance $\epsilon>0$. Let $k=1$;

(2) Compute the dual solutions $\left\{\boldsymbol{\sigma}^{(k)}\right\}$ by applying the SDP solver to problems (4.5), (4.8) and (4.14), respectively.

(3) Compute the primal solution $\mathbf{w}^{(k)}=\left[\mathbf{G}\left(\boldsymbol{\sigma}^{(k)}\right)\right]^{-1} \mathbf{f}$.

(4) To check convergence; if $\left\|\mathbf{w}^{(k)}-\mathbf{w}^{(k-1)}\right\| /\left\|\mathbf{w}^{(k)}\right\| \leq \epsilon$, stop with the optimal solution $\mathbf{w}^{*}=\mathbf{w}^{(k)}$. Otherwise, let $k=k+1$ and go to step 2 .

The SDP solver used in this algorithm is a popular software package named SeDuMi, which is based on the interior point method [33].

\section{Numerical solutions}

We present in this section two different types of beams. Geometrical data were kept fixed for all computations; elastic modulus $E=1000 P a$, Poisson's ratio $\mu=0.3$ and beam length $L=1 \mathrm{~m}$. The lateral load $q(x)$ is assumed to be either a uniformly distributed load such that $f(x)=\left(1-\mu^{2}\right) q(x)=0.1 N / \mathrm{m}$ or a concentrated force on the center of the beam in which $f(x)=0.1 N$. A different number of elements with the same beam length, different compressive load $\lambda$ with different values of beam height are applied in this paper.

5.1. Simply supported beam. A simply supported beam model is fixed in both directions at $x=0$ and fixed only in the y-direction at $x=L$ as shown in Figure (5.1-a) with the boundary conditions $w(0)=w^{\prime \prime}(0)=w(L)=w^{\prime \prime}(L)=0$.

If the beam height is 0.1 (i.e. $h=0.05 \mathrm{~m}$ ), the critical load is $\lambda_{c r}=0.00097 \mathrm{~m}^{2}$ (see Equation (2.6)). For a different number of beam elements, the approximate deflections 


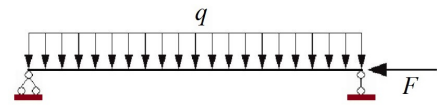

(a) Simply supported beam

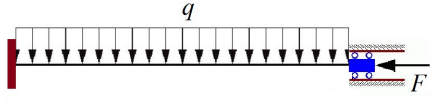

(b) Clamped beam
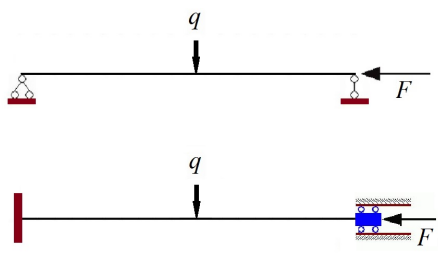

FIG. 5.1. Types of beams - uniformly distributed load (left), concentrated force (right)
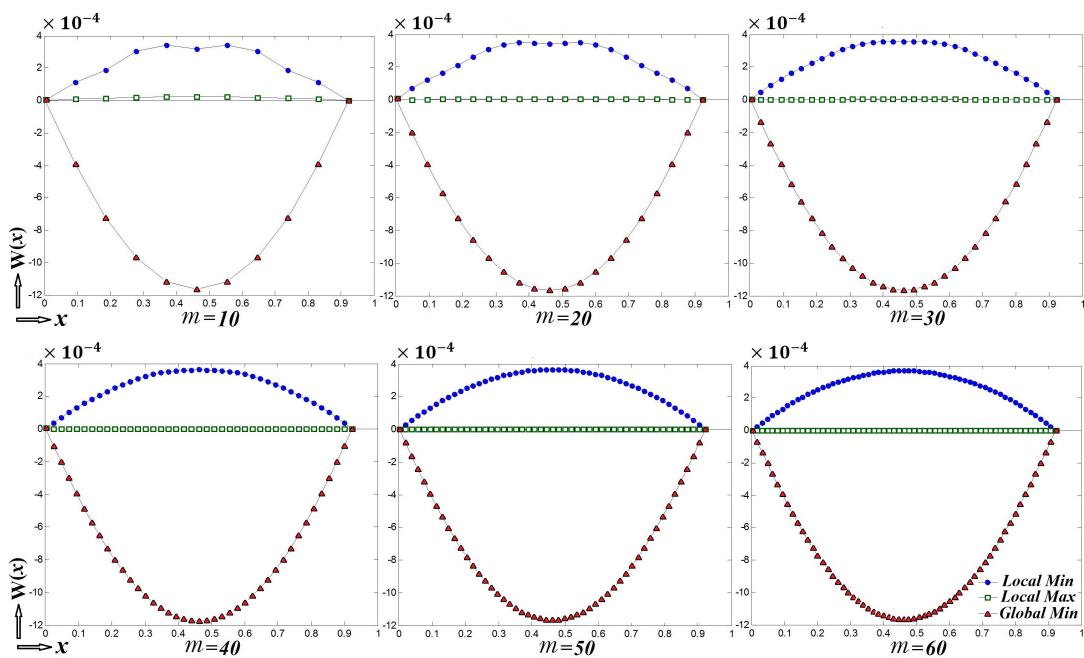

FIG. 5.2. Simply supported beam under a uniformly distributed load with $\lambda=0.01 \mathrm{~m}^{2} \quad(h=0.05 \mathrm{~m})$

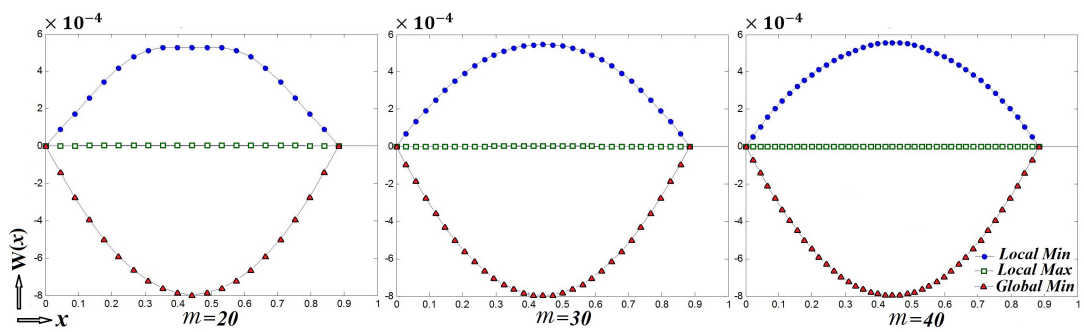

FIG. 5.3. Simply supported beam under a uniformly distributed load with $\lambda=0.015 \mathrm{~m}^{2} \quad(h=0.05 \mathrm{~m})$

of this beam with $\lambda=0.01 \mathrm{~m}^{2}>\lambda_{c r}$ under a uniformly distributed load are illustrated in Figure 5.2. In the graphs, red triangle represents the global minimum, green square represents the local maximum and blue circle represents the local minimum of $\Pi(w)$. Figure 5.2 shows that the two post-buckled configurations; global minimum and local maximum, look alike with all of the different number of beam elements. In contrast to the local minimum, few differences appear in the local unstable buckled configuration. The curve charts with 40,50 and 60 elements seem very similar and more stable than the curve charts that contain 10, 20 and 30 elements. Once again, Figure 5.3 shows that, with a different number of elements at $\lambda=0.015 \mathrm{~m}^{2}>\lambda_{c r}$, slight differences appear on the local minimum curves. 


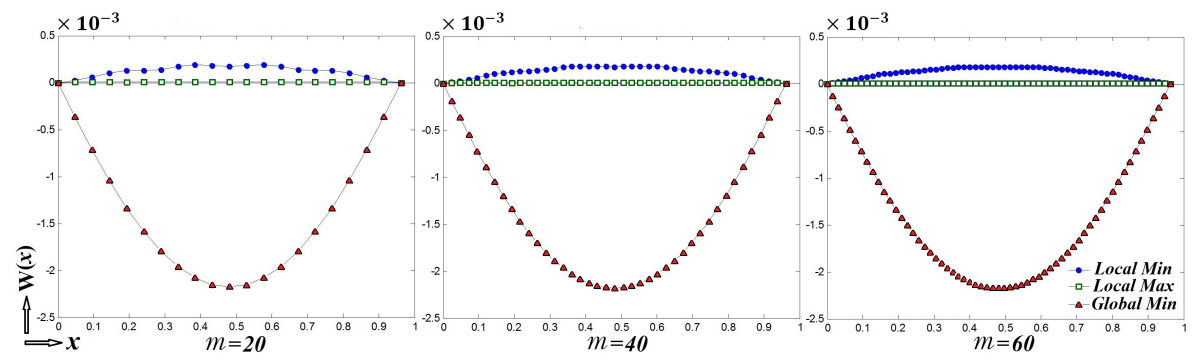

FIG. 5.4. Simply supported beam under a uniformly distributed load with $\lambda=0.005 \mathrm{~m}^{2}(h=0.05 \mathrm{~m})$
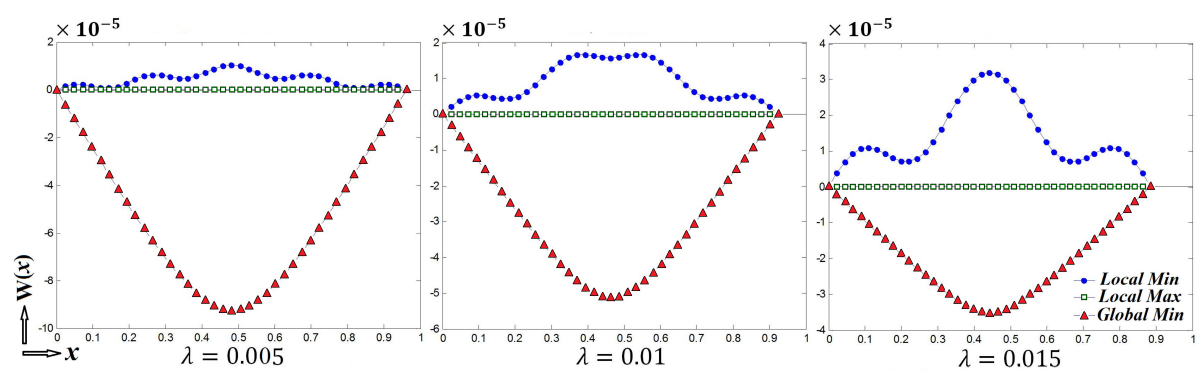

FIG. 5.5. Simply supported beam under a concentrated force $(h=0.05 \mathrm{~m})$
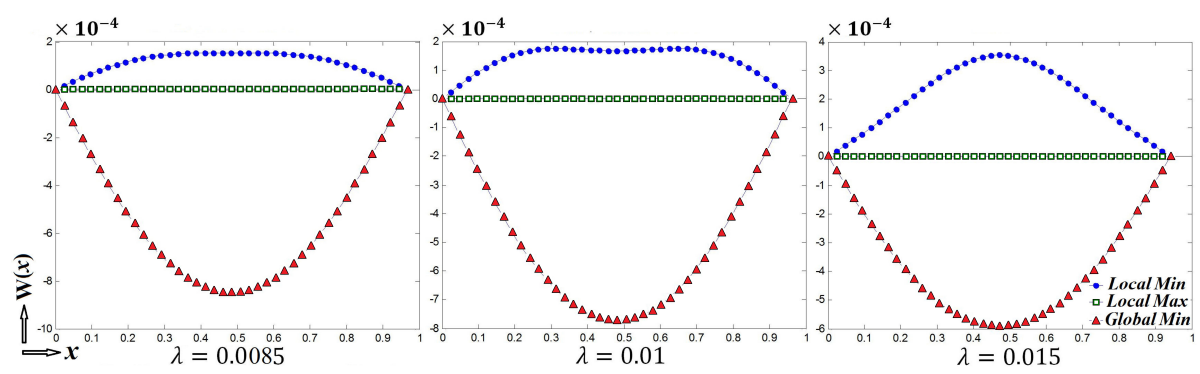

FIG. 5.6. Simply supported beam under a uniformly distributed load $(h=0.1 \mathrm{~m})$

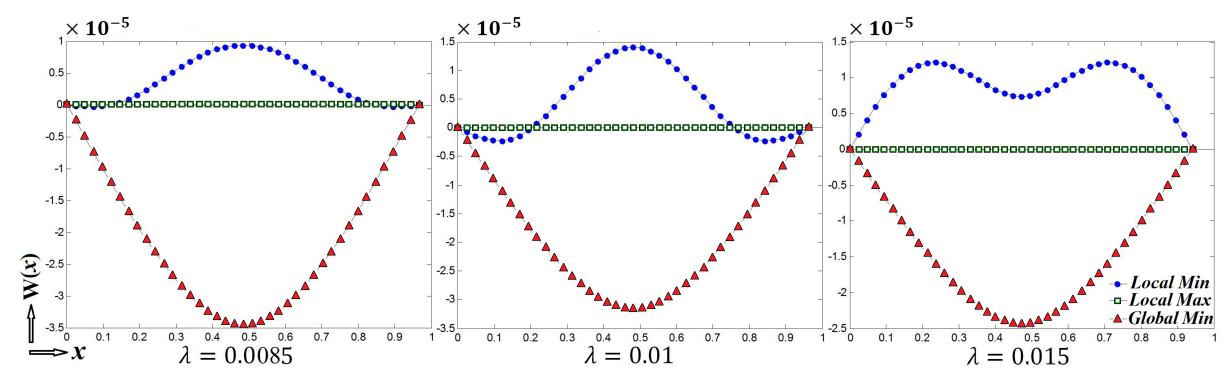

FIG. 5.7. Simply supported beam with a concentrated force $(h=0.1 \mathrm{~m})$

The local minimum solutions with a different number of beam elements at a compressive load $\lambda=0.005 \mathrm{~m}^{2}>\lambda_{c r}$ look alike, as shown in Figure 5.4. The Gao-Strang gap function for all post-buckled solutions was computed under a uniformly distributed load 
for a different number of elements with $\lambda=0.01 \mathrm{~m}^{2}$ as reported in Table 5.1 .

We focus on 40 elements with the same beam length for all the following examples. The deflections of the simply supported beam under a concentrated force with different compressive loads $\lambda>\lambda_{c r}$ are illustrated in Figure 5.5. At $h=0.1 \mathrm{~m}$, the critical load of the simply supported beam is $\lambda_{c r}=0.0078 \mathrm{~m}^{2}$. The deflections of this beam under a uniformly distributed load and a concentrated force are summarized in Figures 5.6 and 5.7, respectively. The Gao-Strang gap function for all three post-buckled solutions was computed under a uniformly distributed load and a concentrated force as reported in Tables 5.2 and 5.3, respectively.

\begin{tabular}{|c||c|l|l|l|}
\hline \multirow{2}{*}{$\begin{array}{c}\text { Compressive } \\
\text { load }\end{array}$} & $\begin{array}{c}\text { No elements } \\
m\end{array}$ & \multicolumn{3}{|c|}{ Gap function under a uniformly distributed load } \\
\cline { 3 - 5 } & & Global Min & Local Min & Local Max \\
\hline \multirow{3}{*}{$\lambda=0.01$} & 20 & $7.63568 \mathrm{e}-09$ & $-2.15332 \mathrm{e}-09$ & $-4.16926 \mathrm{e}-07$ \\
& 40 & $1.45323 \mathrm{e}-09$ & $-8.56515 \mathrm{e}-10$ & $-1.04182 \mathrm{e}-07$ \\
& 60 & $6.10785 \mathrm{e}-10$ & $-4.93895 \mathrm{e}-10$ & $-4.62995 \mathrm{e}-08$ \\
\hline
\end{tabular}

TABLE 5.1. Gao-Strang gap function for simply supported beam with different numbers of elements

\begin{tabular}{|l||l|l|l|l|}
\hline \multirow{3}{*}{ Beam height } & Compressive & \multicolumn{3}{|c|}{ Gap function under a uniformly distributed load } \\
\cline { 3 - 5 } & loads " $\lambda$ " & Global Min & Local Min & Local Max \\
\hline \multirow{3}{*}{$h=0.05$} & 0.005 & $1.38767 \mathrm{e}-09$ & $-3.90449 \mathrm{e}-10$ & $-1.04182 \mathrm{e}-07$ \\
& 0.01 & $1.45323 \mathrm{e}-09$ & $-8.56515 \mathrm{e}-10$ & $-1.04182 \mathrm{e}-07$ \\
& 0.015 & $1.51964 \mathrm{e}-09$ & $-1.01164 \mathrm{e}-09$ & $-1.04182 \mathrm{e}-07$ \\
\hline \multirow{3}{*}{$h=0.1$} & 0.0085 & $1.66885 \mathrm{e}-10$ & $-1.48050 \mathrm{e}-10$ & $-1.30228 \mathrm{e}-08$ \\
& 0.01 & $1.67195 \mathrm{e}-10$ & $-1.50613 \mathrm{e}-10$ & $-1.30228 \mathrm{e}-08$ \\
& 0.015 & $1.68227 \mathrm{e}-10$ & $-1.55455 \mathrm{e}-10$ & $-1.30228 \mathrm{e}-08$ \\
\hline
\end{tabular}

TABLE 5.2. Gao-Strang gap function for simply supported beam under a uniformly distributed load

\begin{tabular}{|l||l|l|l|l|}
\hline \multirow{3}{*}{ Beam height } & Compressive & \multicolumn{3}{|c|}{ Gap function under a concentrated load } \\
\cline { 3 - 5 } & loads " $\lambda$ " & Global Min & Local Min & Local Max \\
\hline \multirow{3}{*}{$h=0.05$} & 0.005 & $2.72407 \mathrm{e}-12$ & $-9.56982 \mathrm{e}-13$ & $-1.89005 \mathrm{e}-10$ \\
& 0.01 & $2.84230 \mathrm{e}-12$ & $-1.78093 \mathrm{e}-12$ & $-1.89005 \mathrm{e}-10$ \\
& 0.015 & $2.96381 \mathrm{e}-12$ & $-2.05556 \mathrm{e}-12$ & $-1.89005 \mathrm{e}-10$ \\
\hline \multirow{3}{*}{$h=0.1$} & 0.0085 & $3.28941 \mathrm{e}-13$ & $-2.95470 \mathrm{e}-13$ & $-2.36257-11$ \\
& 0.01 & $3.29501 \mathrm{e}-13$ & $-3.00014 \mathrm{e}-13$ & $-2.36257 \mathrm{e}-11$ \\
& 0.015 & $3.31372 \mathrm{e}-13$ & $-3.08597 \mathrm{e}-13$ & $-2.36257 \mathrm{e}-11$ \\
\hline
\end{tabular}

TABLE 5.3. Gao-Strang gap function for simply supported beam under a concentrated load

5.2. Doubly/Clamped beam. A clamped beam or doubly/clamped beam model is clamped at both ends as shown in Figure (5.1-b). The boundary conditions are defined as; $w(0)=w^{\prime}(0)=w(L)=w^{\prime}(L)=0$. The Euler buckling load of this beam with $h=0.05 \mathrm{~m}$ is $\lambda_{c r}=0.0041 \mathrm{~m}^{2}$. A different number of beam elements are applied with the same conditions and $\lambda=0.009 \mathrm{~m}^{2}$. We found that the results looked alike for all three post-buckled solutions as shown in Figure 5.8. The results of the deflections 


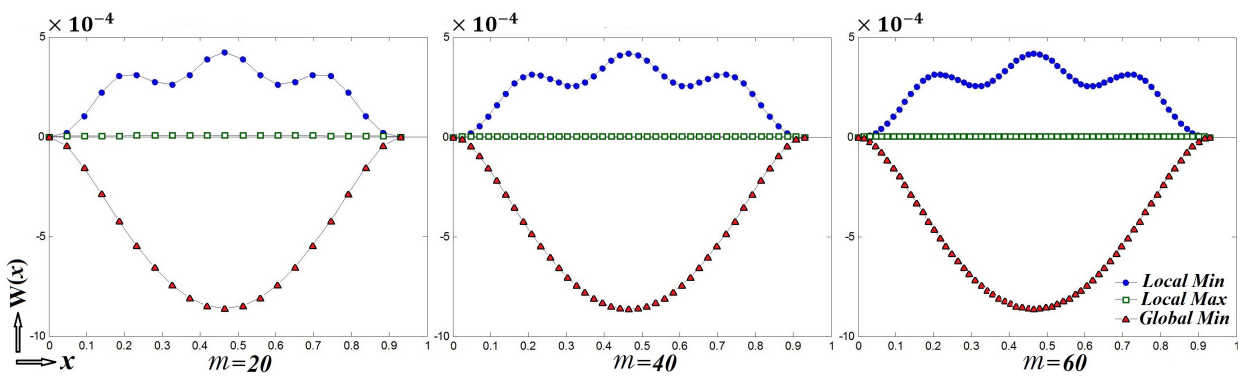

FIG. 5.8. Clamped beam under a uniformly distributed load with $\lambda=0.009 \mathrm{~m}^{2} \quad(h=0.05 \mathrm{~m})$
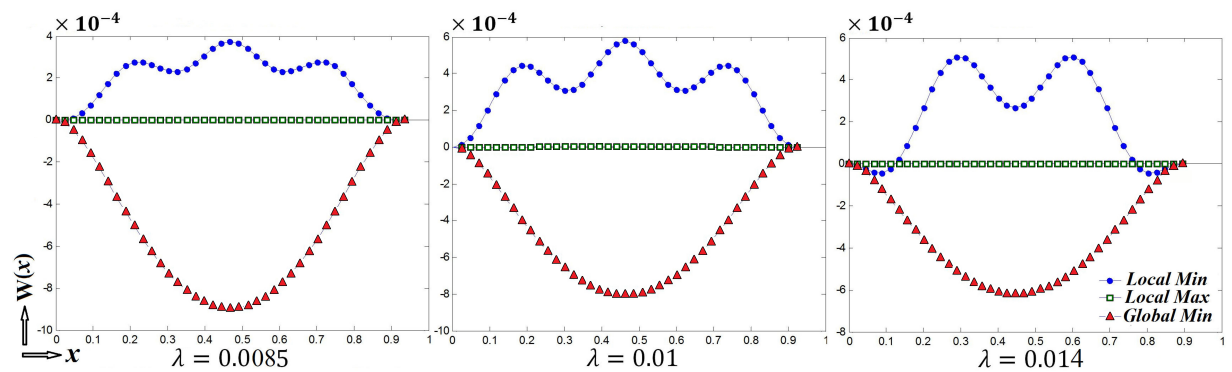

FIG. 5.9. Clamped beam under a uniformly distributed load $(h=0.05 \mathrm{~m})$

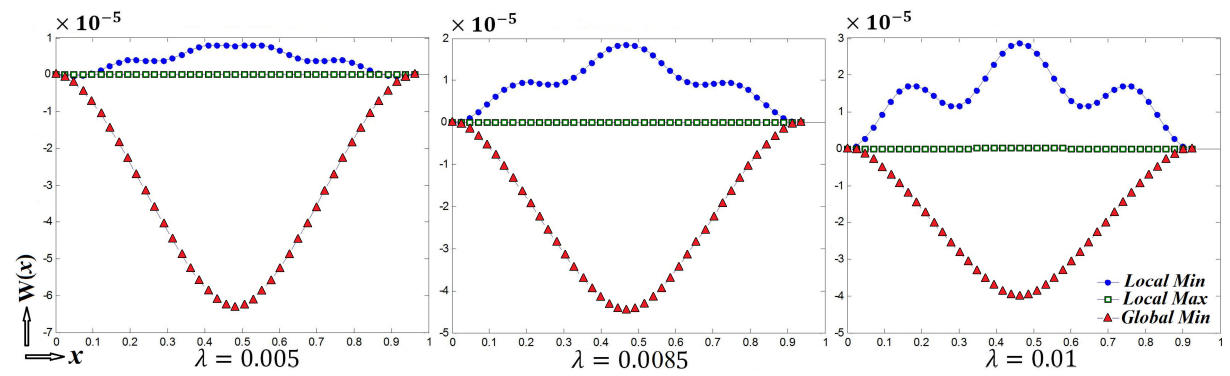

FIG. 5.10. Clamped beam under a concentrated force $(h=0.05 \mathrm{~m})$

\begin{tabular}{|l||l|l|l|l|}
\hline \multirow{3}{*}{ Beam height } & Compressive & \multicolumn{3}{|c|}{ Gap function under a uniformly distributed load } \\
\cline { 3 - 5 } & loads " $\lambda$ " & Global Min & Local Min & Local Max \\
\hline \multirow{3}{*}{$h=0.05$} & 0.0085 & $2.09541 \mathrm{e}-08$ & $-2.01747 \mathrm{e}-08$ & $-1.04101 \mathrm{e}-07$ \\
& 0.009 & $2.09619 \mathrm{e}-08$ & $-2.02106 \mathrm{e}-08$ & $-1.04101 \mathrm{e}-07$ \\
& 0.01 & $2.09768 \mathrm{e}-08$ & $-2.02717 \mathrm{e}-08$ & $-1.04101 \mathrm{e}-07$ \\
& 0.014 & $2.10396 \mathrm{e}-08$ & $-2.04287 \mathrm{e}-08$ & $-1.04101 \mathrm{e}-07$ \\
\hline
\end{tabular}

TABLE 5.4. Gao-Strang gap function for doubly/clamped beam under a uniformly distributed load

under a uniformly distributed load and a concentrated force for different axial loads $\lambda>\lambda_{c r}$ with $m=40$ are illustrated in Figures 5.9 and 5.10, respectively.

The Gao-Strang gap function for all three post-buckled solutions, with different axial loads and beam heights, was computed under a uniformly distributed load and a concentrated force as reported in Tables 5.4 and 5.5, respectively. 


\begin{tabular}{|l||l|l|l|l|}
\hline \multirow{2}{*}{ Beam height } & Compressive & \multicolumn{3}{|c|}{ Gap function under a concentrated load } \\
\cline { 3 - 5 } & loads " $\lambda$ " & Global Min & Local Min & Local Max \\
\hline \multirow{3}{*}{$h=0.05$} & 0.005 & $1.08569 \mathrm{e}-11$ & $-9.01280 \mathrm{e}-12$ & $-1.88954 \mathrm{e}-10$ \\
& 0.0085 & $1.09445 \mathrm{e}-11$ & $-9.72096 \mathrm{e}-12$ & $-1.88954 \mathrm{e}-10$ \\
& 0.01 & $1.09801 \mathrm{e}-11$ & $-9.87268 \mathrm{e}-12$ & $-1.88954 \mathrm{e}-10$ \\
\hline
\end{tabular}

TABLE 5.5. Gao-Strang gap function for doubly/clamped beam under a concentrated load

\section{Conclusions}

We have presented a canonical dual finite element method for the post-buckling analysis of a large deformed elastic beam proposed by Gao in 1996. The nonconvexity of the total potential energy $\Pi(w)$ is necessary for the post-buckling phenomenon, but it leads to a fundamental difficulty for traditional numerical methods and algorithms. Based on the canonical duality theory and mixed finite element method, a new primaldual semi-definite program (PD-SDP) algorithm is proposed, which can be used to solve this challenging nonconvex variational problem to obtain all possible post-buckled solutions. Extensive applications are illustrated for the post-buckled beam with different boundary conditions and axial compressive forces. The Gao-Strang gap function is computed for all post-buckled solutions. It is interesting to note that for local and global minima, the value of this gap function is affected by both, the number of beam elements and axial loads, but for local maxima, its value is affected mainly by the number of elements. Our results show that the number of post-buckling solutions depends mainly on the axial compressive forces. For a given nontrivial $q(x)$, the nonlinear beam can have at most three post-buckled solutions if $\lambda \geq \lambda_{c r}$. Both the global minimizer and local maximizer solutions are very stable. However, the local minimal solution is very sensitive not only to the artificial parameters, such as the size of the finite elements, but also to the natural conditions such as the axial compressive forces and boundary conditions. Particularly, for a given $\lambda>\lambda_{c r}$, the bigger is the external load $q(x)$, the smaller is the local minimal solution $w(x)$. Therefore, the related numerical results presented in Figure 13 in [3] are wrong ${ }^{2}$.

Acknowledgements. The authors would like to sincerely acknowledge the important comments and suggestions from an anonymous reviewer, which significantly improved the quality of the manuscript. This research was supported by the US Air Force Office of Scientific Research under the grants (AOARD) FA2386-16-1-4082 and FA9550-17-1-0151.

\section{REFERENCES}

[1] J. Ahn, K.L. Kuttler, and M. Shillor, Dynamic contact of two Gao beams, Electronic Journal of Differential Equations, 194:1-42, 2012. 2

[2] C.I. Bajer, B. Dyniewicz, and M. Shillor, A Gao beam subjected to a moving inertial point load, Math. Mech. Solids, 23(3):461-472, 2018. 2

[3] K. Cai, D.Y. Gao, and Q.H. Qin, Post-buckling solutions of hyper-elastic beam by canonical dual finite element method, Math. Mech. Solids, 19(6):659-671, 2014. 1, 2, 6, 2

[4] Y. Chen and D.Y. Gao, Global solutions to nonconvex optimization of 4th-order polynomial and log-sum-exp functions, J. Global Optimization, 64(3):417-431, 2016. 3, 3

[5] D.Y. Gao, Canonical duality: Complementary finite-element method for finite deformation nonsmooth mechanics, Journal of Engineering Mathematics, 30:339-353, 1996. 1

\footnotetext{
${ }^{2}$ The first author of [3] is responsible for this mistake since he didn't let the other two co-authors to check his computer code.
} 
[6] D.Y. Gao, Nonlinear elastic beam theory with application in contact problems and variational approaches, Mechanics Research Communications, 23(1):11-17, 1996. 1, 2, 2

[7] D.Y. Gao, Dual extremum principles in finite deformation theory with applications to post-buckling analysis of extended nonlinear beam model, Applied Mechanics Review, 50:S64-S71, 1997. 1

[8] D.Y. Gao, Pure complementary energy principle and triality theory in finite elasticity, Mechanics Research Communications, 26(1):31-37, 1999. 1, 2

[9] D.Y. Gao, General analytic solutions and complementary variational principles for large deformation nonsmooth mechanics, Meccanica, 34:167-196, 1999. 1

[10] D.Y. Gao, Duality Principles in Nonconvex Systems: Theory, Methods and Applications, Springer, New York/Boston, 2000. 2, 2

[11] D.Y. Gao, Canonical duality theory: unified understanding and generalized solutions for global optimization, Comput. \& Chem. Eng., 33(12):1964-1972, 2009. 2

[12] D.Y. Gao, Analytical solutions to general anti-plane shear problems in finite elasticity, Continuum Mech. Thermodyn., 28:175-194, 2016. 1

[13] D.Y. Gao and R.W. Ogden, Multiple solutions to nonconvex variational problems with implications for phase transitions and numerical computation, Quarterly Journal of Mechanics and Applied Mathematics, 61(4):497-522, 2008. 1

[14] D.Y. Gao and R.W. Ogden, Closed-form solutions, extremality and nonsmoothness criteria in a large deformation elasticity problem, Zeitschrift fur angewandte Mathematik und Physik, 59:498-517, 2008. 2

[15] D.Y. Gao, V. Latorre, and N. Ruan, Canonical Duality Theory: Unified Methodlogy for Multidisciplinary Study, Springer, New York, 2017. 1, 2

[16] D.Y. Gao, N. Ruan, and V. Latorre, Canonical duality-triality theory: Bridge between nonconvex analysis/mechanics and global optimization in complex systems, Advances of Canonical Duality-Triality Theory, Spriner, 1-47, 2017. 2

[17] D.Y. Gao and H.D. Sherali, Canonical duality:connection between nonconvex mechanics and global optimization, Advances in Appl. Mathematics and Global Optimization, Springer, 257-326, 2009. 2

[18] D.Y. Gao and G. Strang, Geometric nonlinearity: potential energy, complementary energy and the gap function, Quart. Appl. Math., 47:487-504, 1989. 1, 2, 2, 2

[19] D.Y. Gao and C.Z. Wu, On the triality theory for a quartic polynomial optimization problem, J. Ind. Manag. Optim., 8(1):229-242, 2012. 3, 3

[20] D.Y. Gao and H.F. Yu, Multi-scale modelling and canonical dual finite element method in phase transitions of solids, Int. J. Solids Struct., 45:3660-3673, 2008. 1

[21] K.L. Kuttlera, J. Li, and M. Shillor, Existence for dynamic contact of a stochastic viscoelastic Gao beam, Nonlinear Analysis: Real World Applications, 22:568-580, 2015. 2

[22] K.M. Levere, An inverse problem for the nonlinear Gao beam, Int. J. Applied Nonlinear Science, 1(2):122-135, 2014. 2

[23] S.F. Li and A. Gupta, On dual configurational forces, Journal of Elasticity, 84:13-31, 2006. 1

[24] K.T. Andrews, Y. Dumont, M.F. M'Bengue, J. Purcell, and M. Shillor, Analysis and simulations of a nonlinear elastic dynamic beam, Z. Angew. Math. Phys., 63:1005-1019, 2012. 2

[25] J. Machalová and H. Netuka, Optimal control of system governed by the Gao beam equation, AIMS Proceedings of Dynamical Systems, Differential Equations and Applications, 2015(special):783792, 2015. 2

[26] J. Machalová, H. Netuka, and Hui-Shen, Solution of contact problems for nonlinear Gao beam and obstacle, Journal of Applied Mathematics, 2015:1-12, 2015. 2

[27] J. Machalová and H. Netuka, Control variational method approach to bending and contact problems for Gao beam, Applications of Mathematics, 62(6):661-677, 2017. 2, 2

[28] J. Machalová and H. Netuka, Solution of contact problems for Gao beam and elastic foundation, Math. Mech. Solids, 23(3):473-488, 2018. 2

[29] D.M. Morales and D.Y. Gao, Canonical duality theory and triality for solving general nonconstrained global optimization problems, Mathematics and Mechanics of Complex Systems, 3(2):139-161, 2015. 3, 3

[30] H.A.F.A. Santos and D.Y. Gao, Canonical dual finite element method for solving post-buckling problems of a large deformation elastic beam, International Journal of Non-Linear Mechanics, 47:240-247, 2012. 1, 2

[31] B. Gärtner and J. Matousek, Approximation Algorithms and Semidefinite Programming, Springer Heidelberg Dordrecht London New York, 2012. 4

[32] B. Stephen and V. Lieven, Convex Optimization, Cambridge University Press, First Edition, 2004. 4, 4

[33] J.F. Sturm, Using SeDuMi 1.02, a MATLAB toolbox for optimization over symmetric cones, Optimization Methods and Software, 11:625-653, 1999. 4 